$0.1156 \mathrm{~g}$ Sbst.: $0.1326 \mathrm{~g} \mathrm{CO}_{2}, 0.0390 \mathrm{~g} \mathrm{H}_{3} \mathrm{O} .-0.1295 \mathrm{~g}$ Sbst.: $32 \mathrm{cem}$ N $\left(17^{\circ}, 750 \mathrm{~mm}\right)$.

$\mathrm{C}_{6} \mathrm{H}_{6} \mathrm{O}_{5} \mathrm{~N}_{4}$ (Triamid). Ber. C 33.64, H 2.80, N 26.17.

$\mathrm{C}_{2} \mathrm{H}_{4} \mathrm{O}_{2} \mathrm{~N}_{2}$ (Oxamid). » $\quad 27.27$, " 4.54, "31.31.

Gef. \31.28, 》 3.75 , 28.29 .

Die gefundenen Werthe stimmen mit den für ein Triamid $\mathrm{C}_{6} \mathrm{H}_{6} \mathrm{O}_{5} \mathrm{~N}_{4}$ berechneten, welches wir unter der Voraussetzung, dass die Verbindung $\mathrm{C}_{12} \mathrm{H}_{15} \mathrm{O}_{8} \mathrm{~N}$ ein Tricarbonsăureester sei, erwartet hatten, schlecht überein. Beim Erbitzen sublimirt das Product z. Th. unzersetzt, als ob ibm eine kleine Menge Oxamid beigemengt wäre, auch wird daraus beim Erhitzen mit 40-procentiger Natronlauge eine kleine Menge Oxalsăure gebildet. Durch die Annahme, dass in dem Producte etwas Oxamid enthalten sei, würde die Abweichnng der Analysenzahlen von den für das Triamid $\mathrm{C}_{6} \mathrm{H}_{6} \mathrm{O}_{6} \mathrm{~N}_{4}$ berechneten Werthen erklärlich werden. Wir balten es daher, trotz dem unsicheren analytischen Befunde, für wabrscheinlich, dass das Product im Wesentlichen aus einem Triamid von der Formel $\mathrm{C}_{3} \mathrm{O}_{3} \mathrm{~N}\left(\mathrm{CONH}_{2}\right)_{3}$, somit das Oel $\mathrm{C}_{12} \mathrm{H}_{15} \mathrm{O}_{8} \mathrm{~N}$ aus einem Tricarbonsäureester $\mathrm{C}_{3} \mathrm{O}_{2} \mathrm{~N}\left(\mathrm{COOC}_{2} \mathrm{H}_{5}\right)_{3}$ bestehe. Durch diese Annahme wird die erwähnte Bildungsanalogie des Esters mit dem Trimethylisoxazol, $\mathrm{C}_{3} \mathrm{NO}\left(\mathrm{CH}_{3}\right)_{3}$, aus Nitroäthan wesentlich verschärft, da sie nun in der Zusammensetzung der beiden Verbindungen einen gewissen Parallelismus findet.

140. Richard Möhlau und Max Heinze: Zur Charakteristik der Amidoazoverbindungen.

[Mittheilung aus dem Laboratorium für Farbenchemie und Färbereitechnik der Technischen Hochschule zu Dresden.]

(Eingegangen am 27. März 1901.)

Paraamido- und Orthoamidoazo-Verbindungeu zeigen bekanntlich in mancher Hinsicht nicht unerhebliche Verschiedenheiten, welche dazu geführt baben, für diese beiden Verbindungsgruppen eine abweichende Constitution in Erwaigung zu ziehen.

Während nach den bisherigen Untersuchungen kein zwingender Grund vorliegt, die Paraamidoazokörper anders denn als Amidoverbindungen aufzufassen, bat man sich veranlasst gesehen, die Orthoamidoazokörper zu den tautomeren Verbindungen zu zäblen'), da sie ebensowohl als primäre Amine wie als Hydrazone von Orthochinonimiden zu reagiren vermögen. Die letztere Anschanung ist zuerst von Zincke ${ }^{\text {) }}$ ausgesprochen und experimentell begründet worden.

1) H. Goldschmidt, diese Berichte 23, 498 [1890].

2) Diese Berichto 18, 3132, 3142 [1885].

Berichto d. D. chem. Geaellschaft. Jabrg. XXXIV 
Er -fand, dass die Orthoamidoazoverbindungen durch Verlust zweier Wasserstoffatome in die farblosen Pseudoazimide übergeben, deren Zustandekommen die Chinonformeln leicht erklären lassen. Auch die von H. Goldschmidt und seinen Schülern nachgewiesene Bildung von Triazinderivates ${ }^{1}$ ) bei der Einwirkung von Phenylcyanat und diejenige von Dibydrotriazinverbindungen ${ }^{2}$ ) bei der Einwirkung von Aldebyden lässt sich glatter formuliren, wens man die Ortboamidoazokörper als Chinonderivate aufjasst. Gleiches gilt für eine Reihe neuer Triazinderivate, welche $\mathrm{M} . \mathrm{Busch}{ }^{3}$ ) beim Studium der Einwirkung von Arylsenfölen und Phosgen auf Ortboamidoazokörper erhalten hat.

Auf eine von derjenigen der Paraamidoazokörper verschiedene Reactionsweise der Orthuverbindungen deutet ferner deren Verhalten gegenüber Thionylchlorid hin. Denn während nach A. Michaelis und G. Erdmann') die Paraderivate mit Letzterem stabile Thionylamine liefern, vereinigen sich die Orthoverbindungen darnit nur schwierig. Die Einwirkungsproducte verwandeln sich unter Entwickelung von Schwefeldioxyd leicht in Pseudoazimidokörper.

Dass die Orthoamidoazorerbindungen unzweifelhaft als Amine zu reagiren vermögen, beweist ihre von Noelting und $W_{i t t^{5}}$ ), Zincke und $L$ aw son ${ }^{6}$ ) constatirte Ueberfübrbarkeit in Diazoniumsalze, welche sich insofern allerdings abweichend verhalten, als sie bei geeigneter Reduction nicht Hydrazine, sonderu gegen Reductionsmittel beständige Diazohydrüre oder Isodihydrotetrazine liefern.

Obwobl nun die Paraamidoazoverbindungen sich in allen Reactionen als primäre Amine charakterisiren und keinen Anlass bieten, ihnen Chinonformeln zuzuschreiben, so erschien das Stndium ihres Verhaltens gegenüber Tetramethyldiamidobenzhydrol im Hinblick auf dessen specifische Reactionsweise mit parachinoïden Körpern ${ }^{7}$ ), insbescondere mit Paraoxyazoverbindungen ${ }^{8}$ ), immerhin von Interesse.

$\mathrm{Da}$ Letztere als Chinonhydrazone ein Kernwasserstoffatom durch den Hydrolrest ersetzen lassen, so war eine im gleichen Sinne verlaufende Wecbselwirkung zwischen Paraamidoazokörpern und Tetra-

1) H. Goldschmidt u. Rosell, diese Berichte 23, 501 [1890)

2) H. Goldschmidt u. Rosell, diese Berichte 23, 505 [1890]; H. Goldschmidt u. Poltzer, diese Berichte 24, 1000 [1891]; E. Noelting n. FF.:Wegelin, diese Berichte 30, 2595 [1897]; R. Meldola, Journ. Chem. Soc.57, 328 [1890]; 59, 678 [1891].

3) Diese Berichte 32, 2959 [1899]. ") Diese Berichte 28, 2192 [1895].

5) Diese Berichto 17, 80 [1884].

ด) Diese Berichte 19, 1452 [1886]; 20, 1176, 2896 [1887].

7) R. Möhlau u. V. Klopfer, diese Berichte 31, 2351 [1898]; 32, 2146 [1899].

8) R. Möhlau u. E. Kegel, diese Berichte 33, 2858 [1900]. 
methyldiamidobenzhydrol dabin zu deuten, dass Erstere als Chinonimidarylhydrazone zu reagiren im Stande sind.

Der Versuch hat gezeigt, dass thatsächlich eine Einwirkung erfolgt. Die Unbeständigkeit der neuen Verbindungen gegenüber Säuren erlaubt indessen nicbt, sie als Chinónderivate anzusprechen. In völligem Einklang steht sie jedoch nit deren Auffassung als Leukauramine.

Hiernach sind die Paraamidoazokörper bei dieser Reaction mit einem Amidowasserstoff betheiligt, wie die Gleichung

$\mathrm{C}_{6} \mathrm{H}_{5} \cdot \mathrm{N}_{2} \cdot \mathrm{C}_{6} \mathrm{H}_{4} \cdot \mathrm{NH}_{2}+\mathrm{HO} \cdot \mathrm{CH}\left[\mathrm{C}_{6} \mathrm{H}_{4} \cdot \mathrm{N}\left(\mathrm{CH}_{3}\right)_{2}\right]_{2}$

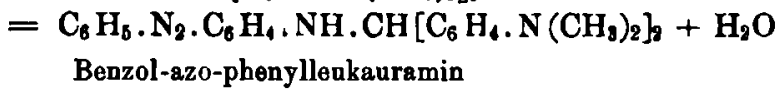

beispielsweise erkennen lässt. Der leichte Zerfall des entstandenen Productes in seine Componenten unter dem Einfluss von Säuren entspricht der Zerlegung des Leukauramins in Ammoniak und Tetramethyldiamidobenzhydrol.

Es erhob sich nun die Frage, wie sich Orthoamidoazokörper diesem Hydrol gegenüber verhalten würden.

Das Ergebniss des Versuchs ist die bemerkenswerthe Thatsache, dass es lediglich von der Temperatur abhingt, ob die Orthoamidoazoverbindungen den Paraderivaten durchaus analog damit reagiren oder nicht.

Wäbrend die Paraamidoazokörper innerbalb weiter Temperatargrenzen ausschliesslich Leukauramine bilden, lassen die Orthoamidoazoverbindungen nur bei mässiger Temperatur (bis $40^{\circ}$ ) Leukauramine, bei höherer Temperatur (bis $80^{\circ}$ ) aber A uramine entstehen.

In diese Auramine wandeln sich daher auch die vorgebildeten Leukauramine bei höherer Temperatur um, z. B.

$\mathrm{C}_{7} \mathrm{H}_{7} \cdot \mathrm{N}_{7} \cdot \mathrm{C}_{7} \mathrm{H}_{6} \cdot \mathrm{NH} \cdot \mathrm{CH}\left[\mathrm{C}_{6} \mathrm{H}_{4} \cdot \mathrm{N}\left(\mathrm{CH}_{3}\right)_{8}\right]_{2}$

$p$-Tolnol-azo-p-tolyllenkauramin

$$
=\mathrm{C}_{7} \mathrm{H}_{7} \cdot \mathrm{N}_{2} \cdot \mathrm{C}_{7} \mathrm{H}_{8} \cdot \mathrm{N}: \mathrm{C}\left[\mathrm{C}_{6} \mathrm{H}_{4} \cdot \mathrm{N}\left(\mathrm{CH}_{3}\right)_{2}\right]_{2}+\mathrm{H}_{2}
$$

$p$-Toluol-azo-p-tolylauramin.

Experimenteller Theil.

I. Condensation von Paraamidoazoverbindungen mit Tetramethyldiamidobenzhydrol.

Die Vereinigung äquimolekularer Mengen eines Paraamidoazokörpers und Michler'schen Hydrols vollzieht sich innerhalb zwölf Stunden schon bei gewöhnlicher Temperatur, weit schneller geht dieselbe bei Wasserbadtemperatur vor sich. 


\section{Zur Darstellung des}

Benzol-azo-phenylleukauramins,

$\mathrm{C}_{6} \mathrm{H}_{5} \cdot \mathrm{N}_{2} \cdot \mathrm{C}_{6} \mathrm{H}_{4} \cdot \mathrm{NH} \cdot \mathrm{CH}\left[\mathrm{C}_{6} \mathrm{H}_{4} \cdot \mathrm{N}\left(\mathrm{CH}_{3}\right)_{2}\right]_{2}$,

wurden $19.7 \mathrm{~g}$ ( $1 \mathrm{Mol}$ ) $p$-A midoazobenzol und $27 \mathrm{~g}$ ( $1 \mathrm{Mol}$.) Michler'sches Hydrol in $120 \mathrm{~g}$ Alkohol von $96 \mathrm{pCt}$. gelöst und gegen fünf Stunden auf dem Wasserbade am Rückflusskühler erbitzt. Das sich krystalliniscls ausscheidende Reactionsproduct wurde mit Alkohol gewaschen und in heissem Benzol aufgenommen. Auf Zusatz von Ligroün schieden sich bräunlicbgelbe Prismen vom Schmp. 156.5 in einer Menge von $36 \mathrm{~g}$, entsprechend $80 \mathrm{pCt}$. der theoretischen Ausbeute, ab.

$0.4474 \mathrm{~g}$ Sbst.: $1.2731 \mathrm{~g} \mathrm{CO}_{2}, 0.2766 \mathrm{~g} \mathrm{H}_{2} \mathrm{O} .-0.3165 \mathrm{~g}$ Sbst.: $42.4 \mathrm{ccm}$ N (150, $746 \mathrm{~mm})$.

$$
\begin{array}{lll}
\dot{\mathrm{C}}_{29} \mathrm{H}_{31} \mathrm{~N}_{3} . & \text { Ber. C } 77.50, \mathrm{H} 6.90, \mathrm{~N} & 15.59 . \\
& \text { Gef. } \gg 77.60, 》 6.87 \text {, \ } 15.43 .
\end{array}
$$

In Alkohol und $\Delta$ ether ist die Verbindung schwer löslich, dagegen leicht löslich in heissem Benzol, Aceton und Chloroform. Concentrirte Salzsäure und Schwefelsäure lōsen sie mit rother bezw. gelber Farbe, Eisessig löst sie mit braunrother Farbe. Die essigeaure Lōsung nimmt beim Erwärmen die blaue Lösungsfarbe des Tetramethyldiamidobenzhydrolacetats an.

In verdünnter Salzsäure und Schwefelsäure ist sie in der Kălte gleichfalls leicht mit intensiv rotber Farbe löslich. Nach längerem Steben, schneller beim Erwärmen, scheidet sich Amidoazobenzolsalı ab, während Tetramethyldiamidobenzbydrol in Lösung bleibt.

Dieses Verbalten kennzeichnet die Verbindung als Benzol-azopheuylleukauramin.

Wird dasselbe der Acetylirung mit Essigsäureanhydrid und Natriumacetat unterworfen, so entsteht Acetyl-p-amidoazobenzol

Dasselbe wurde nach mebrfachem Umkrystallisiren aus Alkohol. in gelben Nadeln rom Schup. $141^{\circ}$ erhalten.

0.2442 g Sbst.: $0.6315 \mathrm{~g} \mathrm{CO}_{2}, 0.126 \mathrm{~g} \mathrm{H}_{2} \mathrm{O}$.

$$
\mathrm{C}_{14} \mathrm{H}_{13} \mathrm{ON}_{3} \text {. } \begin{aligned}
& \text { Ber. C } 70.29 \text {, H } 5.44 \text {. } \\
& \text { Gef. } 70.56, \quad 5.73 \text {. }
\end{aligned}
$$

Mit Dimethylamidoazobenzol vereinigt sich das Michler'sche Hydrol nicht, ein weiterer Beleg dafür, dass bei der in Rede stehenden Reaction der Amidoazokörper Kernwasserstoff nicht betheiligt ist.

$$
\begin{aligned}
& \text { Benzol-azo- } \alpha-n \text { aph tylleukauramin, } \\
& \mathrm{C}_{6} \mathrm{H}_{5} \cdot \mathrm{N}_{2} \cdot \mathrm{C}_{10} \mathrm{H}_{6} \cdot \underset{(\alpha)}{\mathrm{NH}} \mathrm{CH}\left[\mathrm{C}_{6} \mathrm{H}_{4} \cdot \mathrm{N}\left(\mathrm{CH}_{3}\right)_{2}\right]_{3} \text {. }
\end{aligned}
$$

Das in analoger Weise durch fünfstündiges Erbitzen einer alkoholischen Lösung von $24.7 \mathrm{~g}$ ( $1 \mathrm{Mol}$.) Benzol-azo- $\alpha$-naphtylamin und $27 \mathrm{~g}$ ( $1 \mathrm{Mol}$.) Michler'schem Hydrol bereitete and in Form einer rothbraunen Krystallmasse sich abscheidende Benzol-azo- $\alpha$-naphtyl- 
leukauramin ist in Alkohol, Aether und Aceton scbwer, in Chloroform und warmem Benzol dagegen leicht löslich. Aus der Lösung in Chloroform oder Benzol kryatallisirt es auf Zugabe ron Alkohol in gelbbraunen Prismen, welche bei $234-235^{\circ}$ schmelzen. Die Ausbente betrug $31 \mathrm{~g}$ oder $62 \mathrm{pCt}$. der Theorie.

$0.158 \mathrm{~g}$ Sbst.: $0.4595 \mathrm{~g} \mathrm{CO}_{\mathrm{q}} 0.0992 \mathrm{~g} \mathrm{H}_{2} \mathrm{O} .-0.2489 \mathrm{~g}$ Sbst.: $30.6 \mathrm{ccm}$ N $\left(21^{0}, 761 \mathrm{~mm}\right)$.

$$
\begin{array}{ll}
\mathrm{C}_{33} \mathrm{H}_{33} \mathrm{~N}_{5 .} & \text { Ber. C 79.35, H 6.61, N } 14.03 . \\
& \text { Gef. 》 79.31, 6 6.97, " } 14.03 .
\end{array}
$$

Im Gegensatz zum Benzol-azo- $\alpha$-naphtylamin löst sich dessen Leukauraminderivat in verdünnter Salzeăure sebr leicht mit tief violetter Farbe. Die Lösung scheidet nach einiger Zeit, sofort beim Erwärmen grünschillernde Krystalle von salzsaurem Benzol-azonaphtylamin $a b$, das Filtrat nimmt nach dem Uebersättigen mit Natronlauge auf Zugabe von Eisessig die blane Lösungsfarbe des Micbler'schen Hydrols an.

In concentrirter Schwefelsäure löst sich das Benzol-azo-x-naphtylleukauramin mit blauer, in Eisessig mit rothvioletter Farbe, welche beim Erwärmen in Blau umschlägt.

Bei der Acetylirung mit Essigsäureanhydrid und Natriumacetat wird Acetyl-Benzol-azo-a-naphtylamin gebildet, welches aus Alkohol in hellgelben Blăttchen vom Schmp. $233^{\circ}$ krystallisirte.

$0.4566 \mathrm{~g}$ Sbst.: $1.2476 \mathrm{~g} \mathrm{CO}$, $0.2166 \mathrm{~g} \mathrm{H}_{2} \mathrm{O}$.

$$
\begin{array}{ll}
\mathrm{C}_{18} \mathrm{H}_{15} \mathrm{ON}_{3} \text {. } & \text { Ber. C 74.74, H 5.19. } \\
& \text { Gef. 74.51, } 5.26 .
\end{array}
$$

\section{Condensation von Orthoamidoazoverbindungen mit Tetramethyl- diamidobenzhydrol.}

Wie Eingangs ausgeführt wurde, unterscheiden sich die Orthoamidokörper von den Paraverbindungen in ihrer Reactionsweise mit Michler'schem Hydrol insofern wesentlich, als sie nur bei mässiger Temperatur Leukaramine bilden, bei Wasserbadtemperatur aber Auramine entstehen lassen. Die Ausbeute an Letzteren lässt zu wünschen übrig, sie wird beeinträchtigt durch den aus der Leukauraminverbinduog abgelösten $W$ asserstoff, welcher zur reducirenden Spaltang eines Theils des Azoauraminkörpers verwendet wird.

$$
\begin{gathered}
p \text {-Toluol-azo-p-tolylleukanramin, } \\
\underset{(4)}{\mathrm{CH}_{3}} \cdot \mathrm{C}_{6} \mathrm{H}_{4} \cdot \underset{(1)}{\mathrm{N}_{2}} \cdot \mathrm{C}_{6} \mathrm{H}_{3}\left(\underset{(4)}{\mathrm{CH}_{3}}\right) \cdot \underset{(2)}{\mathrm{NH}} \cdot \mathrm{CH}\left[\mathrm{C}_{6} \mathrm{H}_{4} \cdot \mathrm{N}\left(\mathrm{CH}_{3}\right)_{2}\right]_{8} \cdot
\end{gathered}
$$
(4)
(1)
(4)

$\mathrm{Zu}$ dessen Gewinnung wurde die warm bereitete Lösung von $15.7 \mathrm{~g}$ (1 Mol.) o-Azo-p-amidotoluol und $19 \mathrm{~g}$ ( $1 \mathrm{Mol}$.) Michler'schem Hydrol in $100 \mathrm{~g}$ Alkohol wäbrend $\mathrm{z}$ wölf Stunden bei gewöhnlicher Temperatur unter zeitweiligem Scbütteln sich selbst überlassen. Er- 
wärmt man hierauf noch kurze Zeit bis auf höchstens $40^{\circ}$, so nimmt die krystallinische Ausscheidung noch etwas zu. Die abgesaugten und wit Alkobol gewaschenen, rothen Krystalle wogen trocken $20 \mathrm{~g}$. Dies entspricht einer Ausbeute von $87 \mathrm{pCt}$. der Theorie.

Am schönsten krystallisirt dieses Leukauramin aus Benzol auf Zusatz von etwas Petroläther und $z$ war in rubinrothen Nadeln vom Schmp. $174.5^{\circ}$.

$0.213 \mathrm{~g}$ Sbst.: $0.6102 \mathrm{~g} \mathrm{CO}_{2}, 0.1465 \mathrm{~g} \mathrm{H}_{2}$ 0. $-0.3145 \mathrm{~g}$ Sbst.: $41.6 \mathrm{ccm}$ $\mathrm{N}\left(22^{\circ}, 752 \mathrm{~mm}\right)$.

$$
\begin{aligned}
& \mathrm{C}_{81} \mathrm{H}_{36} \mathrm{~N}_{5} \text {. Ber. C 77.88, H 7.33, N } 14.67 \text {. } \\
& \text { Gef. \78.13, 》 7.64, 》 } 14.82 \text {. }
\end{aligned}
$$

Die Verbindung ist in Alkohol und Aether sehwer löslich, etwas leichter in Aceton, sehr leicht löslich in Chloroform und heissem Benzol. Durch verdünnte Mineralsăuren wird sie theilweise schon bei gewöhnlicher Temperatur, vollständig beim Erwärmen in o-Azop-amidotoluol und Hydrol gespalten, welche als solche isolirt and an ihren Eigenschaften erkannt wurden.

In Eisessig löst sie sich in der Kälte aus dem gleichen Grunde mit blauer Farbe, in concentrirter Schwefelsäure mit orangegelber Farbe, die beim Verdünnen der Lösung mit Wasser in Grūn umschlăgt.

$$
\begin{gathered}
p \text {-Toluol-azo-p-toly lauramin, } \\
\mathrm{CH}_{8} \cdot \mathrm{C}_{6} \mathrm{H}_{4} \cdot \mathrm{N}_{2} \cdot \mathrm{C}_{6} \mathrm{H}_{3}\left(\mathrm{CH}_{3}\right) \cdot \mathrm{N}: \mathrm{C}\left[\mathrm{C}_{6} \mathrm{H}_{4} \cdot \mathrm{N}\left(\mathrm{CH}_{3}\right)_{2}\right]_{2} \text {. }
\end{gathered}
$$

(4) (1) (4) (2)

Wird das $p$-Toluol-azo- $y$-tolylleukauramin mit Alkohol bis zur völligen Lösung gekocht, so ist in einer Probe mit Eisessig kein Hydrol mebr vachweisbar, die Flüssigkeit bleibt gelbroth. Das Leuksuramin ist nun in das Auramin übergegangen.

Nach Verdampfen des Alkohols ist in dem Rückstand Paratoluidin als Product einer tiefgebenden Reduction durch den Geruch leicht nachweisbar. Wird der Rückstand in Benzol gelöst, so fallen auf Zusatz von Petroläther zunächst Schmieren aus, von welchem ubfiltrirt wurde. Das Filtrat schied nacb einiger Zeit orangegelbe Blättchen des Auraminderivates rom Schmp. $190^{\circ}$ in einer Menge von 50 pCt. der angewendeten Leukauraminverbinduug ab.

Dasselbe Product wird aus o-Azo-p-amidotoluol und Michlerschem Hydrol direct durch längeres Kochen in alkoholischer Lösung auf dem Wasserbade erbalten.

$9 \mathrm{~g}$ (1 Mol.) o-Azo-p-amidotoluol und $10.8 \mathrm{~g}$ (1 Mol.) Michlersches Hydrol wurden in $60 \mathrm{~g}$ Alkobol von $96 \mathrm{pCt}$. gelöst und ungefähr fünf Stunden lang am Rückflusskühler erhitzt, bis die Flüssigkeit zu stossen begann. Sie wurde nun schnell gekühlt, da sich bei fortgesetztem Kochen das bereits abgeschiedene Product unter Zersetzung wieder löste. Das Gewicht der abgesaugten, mit Alkohol 
gewaschenen und getrockneten Krystalle betrug $8.5 \mathrm{~g}$ entsprechend $40 \mathrm{pCt}$. der theoretischen Ausbeute. Nach dem Umkrystallisiren aus einem Gemisch von Benzol and Petroläther schmolzen sie gleichfalls bei $190^{\circ}$.

$0.1986 \mathrm{~g}$ Sbst.: $0.5697 \mathrm{~g} \mathrm{CO}_{2}, 0.1306 \mathrm{~g} \mathrm{H}_{2} \mathrm{O} .-0.3547 \mathrm{~g}$ Sbst.: $46.4 \mathrm{ccm}$ N $\left(20^{\circ}, 754 \mathrm{~mm}\right)$.

$$
\begin{aligned}
& \mathrm{C}_{31} \mathrm{H}_{33} \mathrm{~N}_{5} . \quad \text { Ber. C 78.31, H 6.94, N } 14.73 . \\
& \text { Gef. » 78.23, } 7.30, \gg 14.83 .
\end{aligned}
$$

Die Verbindnng ist in Alkohol, Aether und Ligroïn schwer, in Benzol, Aceton und Chloroform leicht löslich.

Als Auramin wird sie durch Mineralsäuren in o-Azo-p-amidotoluol nnd Tetramethyldiamidobenzophenon gespalten. Die tief rothe Lösung in concentrirter Salzeäure verblasst beim Erwärmen schnell und scheidet alsbald das salzsaure Salz des Azokörpers in blassgelben Nadeln ab. Die aus Alkohol umgelöste orangerothe Base schmolz bei $118^{\circ}$. Die aus dem Filtrat mit Alkali gefällte Ketonbase krystallisirte aus Alkohol in Blättchen vom Schmp. 1730.

Dieser leichten Zersetzlichkeit halber waren Salze nicht darstellbar. Eisessig nimmt die Auramiubase mit gelbrother, concentrirte Schwefelsäure mit gelber Farbe auf. Ihre mit Natriumamalgam behandelte alkobolische Lösung wird in Folge von Leukauraminbildung durch Eisessig blan gefärbt.

$$
\begin{gathered}
\text { Benzol-azo- } \beta \text {-naphtylleukauramin, } \\
\mathrm{C}_{6} \mathrm{H}_{5} \cdot \underset{(1)}{\mathrm{N}_{2}} \cdot \mathrm{C}_{10} \mathrm{H}_{6} \cdot \underset{(2)}{\mathrm{NH}} \mathrm{CH}\left[\mathrm{C}_{6} \mathrm{H}_{4} \cdot \mathrm{N}\left(\mathrm{CH}_{3}\right)_{2}\right]_{2} .
\end{gathered}
$$

Die Condensation von Benzol-azo-p-naphtylamin mit Michlerschem Hydrol zum Leukauraminderivat vollzieht sich in alkoholischer Lösung bei gewöhnlicher Temperatur in etwa zwölf Stunden. Sie wird durch zeitweises Erwärmen bis auf $40^{\circ}$ und bäufiges Durchschütteln der Flüssigkeit wesentlich gefördert, wie die Zunahme der sich abscheidenden Krystalle deutlich erkennen lässt. Die Lösung von $24.7 \mathrm{~g}$ ( $1 \mathrm{Mol}$.) Benzol-azo- $\beta$-naphtylamin und $27 \mathrm{~g}$ (1 Mol.) Michler'schem Hydrol in $270 \mathrm{~g}$ Alkobol lieferte $45 \mathrm{~g}$ mit Alkobol gewaschenes und getrocknetes Rohproduct, entsprechend einer lusbeute ron $90 \mathrm{pCt}$. der Theorie.

Aus heissem Aceton krystallisirt der Körper in scharlachrothen Nadeln vom Schmp. $184^{\circ}$.

$0.1566 \mathrm{~g}$ Sbst.: $0.4545 \mathrm{~g} \mathrm{CO}_{2}, 0.0979 \mathrm{~g} \mathrm{H}_{2} \mathrm{O}-0.1031 \mathrm{~g}$ Sbst.: $13.0 \mathrm{c} \mathrm{cm}$ $\mathrm{N}\left(21^{\circ}, 746 \mathrm{~mm}\right)$.

$$
\begin{aligned}
& \mathrm{C}_{33} \mathrm{H}_{33} \mathrm{~N}_{5} \text {. Ber. C 79.36, } \mathrm{H} \text { 6.61, N 14.03. } \\
& \text { Gef. \79.15, 》6.94, 》14.09. }
\end{aligned}
$$

Er ist in Alkohol und Aether schwer, in Benzol, Chloroform und Aceton leicht löslich. Eisessig löst ihn mit in dicker Schich' rother, in dünner grünlichblauer Farbe, welche beim Erwärmen ief blan 
wird. Die durch diesen Farbenumschlag gekennzeichnete leichte Spaltung in Tetramethyldiamidobenzhydrol und Benzol-azo- $\beta$-naphtylamin bewirken mässig verdünnte Mineralsäuren, welche das Leukauraminderivat mit gelbrother Farbe lösen, unter Abscheidung des Azokörpers schnell in der Wärme. Concentrirte Schwefelsăure nimmt es mit blauvioletter Farbe auf.

Die Umwandlung in die wasserstoffärmere

$$
\text { Verbindung } \mathrm{C}_{33} \mathrm{H}_{31} \mathrm{~N}_{5}
$$

hat sich rollzogen, wenn die Suspension des Benzol-azo- $\beta$-naphtylleukauramins in der siebenfachen Gewichtsmenge Alkohol am Rückflusskübler auf dem Wasserbade so lange gekocht wird, bis völlige Lösung eingetreten ist und eine mit Eisessig versetzte Probe beim Erwärmen sich weder blau noch grün färbt, sondern braunroth bleibt.

Das durch Eindunsten der Lösung gewonnene Product bildete nach dem $W$ aschen mit Alkohol und Trocknen ein dunkel gelbrothes. krystallinisches Pulver, welches in den meisten organischen Solventien, insbesondere in Aether, Essigester, Aceton, Alkobol, Benzol, Chloroform leicht löslich ist, aber aus keinem derselben in gut krystallisirtem Zustande erbalten wurde. Für die Analyse wurde es im Exsiccator bis zur Gewichtsconstanz getrockuet.

$0.167 \mathrm{~g}$ Sbst.: $0.4872 \mathrm{~g} \mathrm{CO}_{2}, 0.0983 \mathrm{~g} \mathrm{H}_{2} \mathrm{O} .-0.2988 \mathrm{~g}$ Sbst.: $36.4 \mathrm{ccm}$ $\mathrm{N}\left(18^{0}, 754 \mathrm{~mm}\right)$.

$$
\begin{aligned}
& \mathrm{C}_{33} \mathrm{H}_{31} \mathrm{~N}_{5} \text {. Ber. C 79.68, H 6.24, N 14.08. } \\
& \text { Gef. 》 79.56, 》 6.54, 》 } 13.96 \text {. }
\end{aligned}
$$

Dic Verbindang schmilzt bei $74^{\circ}$. Sie wird von Eisessig mit braunrother Farbe gelöst, die sich beim Kochen nicht ändert. Auch gegenüber mässig rerdünnter Salzsäure, die sie mit gelbrotber Farbe leicht aufnimmt, erweist sie sich bei kurzem Kochen beständig, bei längerem Erhitzen lässt die Intensität der Färbung etwas nach, doch gelang es nicht, Tetramethyldiamidobenzophenon aufzufinden.

Dieser Umstand macht es zweifelhaft, ob in der Verbindung das

$$
\begin{aligned}
& \mathrm{C}_{6} \mathrm{H}_{5} \cdot \mathrm{N}_{2} \cdot \mathrm{C}_{1}, \mathrm{H}_{6} \cdot \mathrm{N}: \mathrm{C}\left[\mathrm{C}_{6} \mathrm{H}_{4} \cdot \mathrm{N}\left(\mathrm{CH}_{3}\right)_{2}\right]_{2} \\
& \text { Benzol-azo- } \beta \text {-naphtylauramin }
\end{aligned}
$$

vorliegt und ob sie nicht vielmehr als

$$
\mathrm{C}_{10} \mathrm{H}_{6} \gtrless_{\mathrm{N} . \dot{\mathrm{C}}}^{\left.\mathrm{N} . \mathrm{N} . \mathrm{C}_{6} \mathrm{C}_{6} \mathrm{H}_{4} \cdot \mathrm{N}\left(\mathrm{CH}_{3}\right)_{2}\right]_{2}}
$$

Tetramethyldiamidotriphenyldihydronaphto- $\alpha$-triazin

anzusprechen ist. Diese Frage ist noch eingehender zu prüfen.

Beim Kochen eines alkoholischen Lösungsgemisches molekularer Mengen von Benzol-azo- $\beta$-naphtylamin und Michler'schem Hydrol, bis Letzteres mit Essigsäure nicht mehr nachweisbar ist. entsteht die gleiche Verbindung. Ihre Isolirung und Reinigung ist in Folge gleichzeitiger Bildung von Anilin und anderen Nebenproducten umständlich, für ihre Darstellung ist dieses Verfahren nicht geeignet. 